\section{THU0553 LOW ALKALINE PHOSPHATASE LEVELS: COULD IT BE HYPOPHOSPHATASIA?}

C. Tornero ${ }^{1}$, P. Aguado ${ }^{1}$, S. García ${ }^{1}$, J. Tenorio ${ }^{2}$, P. Lapunzina ${ }^{2}$, A. Buño ${ }^{3}$, J.M. Iturzaeta ${ }^{3}$, C. Plasencia ${ }^{1}$, I. Monjo ${ }^{1}, A$. Balsa ${ }^{1} .{ }^{1}$ Rheumatology; ${ }^{2}$ Genetics; ${ }^{3}$ Clinical Laboratory, la Paz University Hospital, Madrid, Spain

Background: Hypophosphatasia (HPP) is a rare inherited disorder of bone and mineral metabolism, caused by mutations in the ALPL (alkaline phosphatase liver type) gene, with reduction of activity of the tissue-non-specific isoenzyme of ALP (TNSALP). The clinical presentation is variable and adult forms of the disease are usually milder than those affecting infants and children, easily overlooked or misdiagnosed, which can lead to erroneous therapeutic decisions.

Objectives: The primary objectives of our study are to estimate the prevalence of patients with adult forms of HPP in a group of patients with persistent hypophosphatasemia, to analyze their clinical and functional characteristics and to compare these findings between those presenting or not these mutations.

Methods: In this Cross-sectional study, 1.536.711 ALP measurements owing to 386.356 patients were evaluated during a six-year period (2009-2015). Patients having at least two values below $35 \mathrm{IU} / \mathrm{l}$ and none above $45 \mathrm{IU} / \mathrm{l}$ constituted the study population. In total, 427 patients were included. Among them, 31 patients were excluded because of presenting causes of secondary HPP and 13 because of lost to follow-up. 108 patients were contacted by phone to fulfill a questionnaire about manifestations related to HPP and health assesment and in order to obtain blood samples to perform the genetic test.

Results: Demographic and clinical characteristics of both groups are shown in Table 1. Of the 108 patients evaluated, the genetic test results of 39 patients are available at this moment (the rest of the results are currently pending). 59\% (23/39) tested positive for the genetic mutation.Despite data are still partial and although the results did not achieve statistical significance, we observed with a greater relevance a higher proportion of patients with HPP presenting with chronic bone pain $(48,7 \%$ vs $25,6 \%, p=0,157)$, muscle weakness $(15,4 \%$ vs $2,6 \%, p=0,112)$ and more necessity of analgesic medication $(p=0,107)$ in patients with HPP in comparison with the control group. Furthermore, there was a non-significant trend in the HPP-group to present dental abnormalities and premature dental loss $(7,6 \%)$, absent manifestations in the control group $(\mathrm{p}=0,123)$. In addition, orthopedic surgery was more common in the HPP group $(12,8 \%)$ compared with the other group $(0 \%)$, $p=0,04$. The prevalence of stress fractures was also higher in patients with HPP $(7,69 \%)$. No significant differences were found in demographic characteristics, vertebral fractures, calcific periarthritis, condrocalcinosis, or FRAX index. In terms of biochemical tests, serum phosphate levels were higher in the HPP group $(4,18$ $\mathrm{mg} / \mathrm{dl})$ in relation to the control group $(3,52 \mathrm{mg} / \mathrm{dl}), \mathrm{p}=0,05$. No differences were

\begin{tabular}{|c|c|c|c|}
\hline Pls Chacacteristica & $\begin{array}{c}\text { HPP group } \\
\mathbf{N}=\mathbf{2 3}\end{array}$ & $\begin{array}{c}\text { Control group } \\
\mathrm{N}=16\end{array}$ & P yalue \\
\hline \multicolumn{4}{|c|}{ Demographic characteristics } \\
\hline Mean age \pm DS & $52,78 \pm 13,28$ & $49,13 \pm 10,70$ & $\mathrm{p}=0,366$ \\
\hline $\begin{array}{l}\text { Sex } \\
\text { - Male } \\
\text { - Eemale } \\
\end{array}$ & $\begin{array}{l}10 / 35(25,6 \%) \\
13 / 39(33,3 \%)\end{array}$ & \begin{tabular}{|l}
$4 / 39(10,2 \%)$ \\
$12 / 39(30,7 \%)$ \\
\end{tabular} & $p=0,236$ \\
\hline $\begin{array}{l}\text { Race } \\
\text { - Caucasic } \\
\text { Black } \\
\end{array}$ & $\begin{array}{l}22 / 39(56,4 \%) \\
1 / 39(2,56 \%)\end{array}$ & $\begin{array}{l}16 / 39(41 \%) \\
0 / 39(0 \%)\end{array}$ & $p=0,398$ \\
\hline \multicolumn{4}{|c|}{ Clinical characteristics } \\
\hline Chronic bəne paiz, n/N (\%) & $19 / 39(48,7 \%)$ & $10 / 39(25,6 \%)$ & $P=0,157$ \\
\hline Muscle weakness, n/N \%) & $6 / 39(15,4 \%)$ & $1 / 39(2,6 \%)$ & $P=0,112$ \\
\hline Calcific periarthritis, $\mathrm{n} / \mathrm{N}(\%)$ & $4 / 39(10,25 \%)$ & $1 / 39(2,56 \%)$ & $=0,282$ \\
\hline Ccondrocalcinosis n/N (\%) & $2 / 39(5,12 \%)$ & $0 / 39(0 \%)$ & $\mathrm{b}=0,215$ \\
\hline Raticnts pecsentig stress fractures, $n / \mathrm{N}(\%)$ & $3 / 39(7,69 \%)$ & $0 / 39(0 \%)$ & \\
\hline Vertebral fractures $(\mathrm{n} / \mathrm{N})(\%)$ & $2 / 39: 5,12 \pi)$ & $0 / 39(0 \%)$ & $2-0,193$ \\
\hline Dental abnormalities anc teeth loss. $\mathrm{n} / \mathrm{N}(\%)$ & $3 / 39(7,69 \%)$ & $0 / 39(0 \%)$ & $P=0,123$ \\
\hline History of erthopedic surgery & $5 / 39(12,8 \%)$ & $0 / 39(0 \%)$ & $\mathrm{P}=0,048$ \\
\hline Use of analgesic medication for pain, $\mathrm{n} / \mathrm{N}(\%)$ & $16 / 39(41,03 \%)$ & $7 / 39(17,9 \%)$ & $\mathrm{P}=0,107$ \\
\hline History of kidney disease & $5 / 39(12,8 \%)$ & $2 / 39(5,12 \%)$ & $\mathrm{P}=0,384$ \\
\hline \multicolumn{4}{|c|}{ Biochemical data } \\
\hline Alkaline phesphatase \pm DS & $21,6 \pm 6,76$ & $29,69 \pm 3,44$ & $P=0,0001$ \\
\hline Serum calcium \pm DS & $9,34 \pm 0,4$ & $9,34 \pm 0,4$ & $P=0,994$ \\
\hline Urine salcium $\pm \mathrm{DS}$ & $61,47 \pm 51,3$ & & \\
\hline Serum phosphate \pm DS & $4,18 \pm 9,91$ & $3,52 \pm 0,48$ & $P=0,05$ \\
\hline Urine phosphorus \pm DS & $341 \pm 433$ & & \\
\hline \multicolumn{4}{|c|}{ Quality of life assessment } \\
\hline VAS pain \pm DS & $3,86 \pm 2,5$ & $2,71 \pm 3,1$ & $P=0,221$ \\
\hline $\mathrm{HAQ}-\mathrm{DI} \pm \mathrm{DS}$ & $0,23 \pm 0,36$ & $0,2 \pm 0,43$ & $P=0,764$ \\
\hline
\end{tabular}

observed neither in pain assessment, measured with the Visual Analogue Scale nor in the Health Assessment Questionaire for disability between both groups. Conclusions: The diagnosis of HPP can be difficult and is often missed or delayed, particularly in adults. The prevalence of HPP between patients with persistent low ALP is high and although the clinical presentation is milder in adults, it often presents with chronic bone pain, weakness, stress fractures and dental abnormalities. These data should promote a more proactive attitude towards detection of adult HPP.

Disclosure of Interest: None declared

DOI: 10.1136/annrheumdis-2017-eular.5547

\section{THU0554 IMMUNOGLOBULIN G4-RELATED DISEASE IN HONG KONG - CLINICAL FEATURES, TREATMENT PRACTICES AND ASSOCIATIONS}

P.H. Li, C. Ho, C.-S. Lau. Department of Medicine, Queen Mary Hospital, Hong Kong, Hong Kong

Background: Immunoglobulin (Ig) G4-related disease (IgG4-RD) is a systemic immune-mediated disease unifying what were previously considered to be unrelated individual organ disorders. The diagnosis and treatment of this characteristic fibroinflammatory condition continues to evolve, but generally remains an underrecognised disease. Local data outside Japanese and Caucasian populations is lacking and few studies have examined factors to predict disease severity or disease prognostication.

Objectives: We conducted this study to review the clinical features, treatment practices and factors associated with more extensive disease involvement in Hong Kong

Methods: We retrospectively evaluated all patients with IgG4-RD over the past 13 years in our centre and combined this with patient data extracted from previous local publications. We analysed the clinical features, treatment practices and factors associated with the number of organ systems involved.

Results: One hundred and four patients ( 55 from our centre and 49 from literature review) were identified. Patients were predominantly older men (mean age 61.9 years, male:female ratio $3: 1)$. Hepatobiliary and pancreatic $(40.4 \%)$, salivary gland (33.6\%), lymph nodes $(29.8 \%)$ and eye $(19.2 \%)$ were the most common systems involved. Lymphadenopathy was associated with glucocorticoid use $(\mathrm{OR}=2.65$, $\mathrm{p}=0.034$ ). Over $90 \%$ of patients had a serum greater than $135 \mathrm{mg} / \mathrm{dl}$ and a IgG4/total IgG ratio greater than $8 \%$. Pre-treatment serum IgG4 levels correlated with the number of organ systems involved $(\beta=0.347, p=0.004)$, and specifically with salivary gland involvement (mean $1109 \mathrm{mg} / \mathrm{dl}$ vs. $599 \mathrm{mg} / \mathrm{dl}, \mathrm{p}=0.012$ ).

Conclusions: We identified pre-treatment serum IgG4-RD to be associated with multi-system disease, especially with salivary gland involvement, highlighting the potential for its use in disease prognostication and monitoring. The reason for this particular correlation remains uncertain but highlights the importance of screening for salivary gland involvement in all IgG4-RD patients, especially in the presence of higher serum IgG4 levels. We also describe the clinical features and treatment modalities of the largest cohort of IgG4-RD in Hong Kong thus far. Increased physician awareness and multidisciplinary efforts are required for optimal management of this masquerading disease. Future studies, especially focusing on treatment strategies within the contexts of different epidemiology and patient characteristics, are urgently needed.

Disclosure of Interest: None declared

DOI: 10.1136/annrheumdis-2017-eular.1120

\section{THU0555 THE RELIABILITY OF TWO VASCULITIS ACTIVITY SCORES (BIRMINGHAM VASCULITIS ACTIVITY SCORE AND BEHCET'S DISEASE CURRENT ACTIVITY FORM2006) IN ANTICIPATING DAMAGE IN A GROUP OF PATIENTS DIAGNOSED WITH BEHCET'S DISEASE}

C.G. Buzatu, S.E. Daia-lliescu, I. Saulescu, A. Borangiu, T. Gudu, V. Bojinca, D. Predeteanu, A. Balanescu, R. Ionescu, D. Opris-Belinski. Internal Medicine and Rheumatology, "Saint Mary" Clinical Hospital, Bucharest, Romania

Background: Validated disease activity scores and damage measurements were developed over time in order to allow a better way to evaluate patients and decide treatment plans.There are scores designed for a great variety of vasculitis like Birmingham Activity Score and others that are more specific like Behcet's Disease Current Activity Form2006.

Objectives: To evaluate the ability of the activity scores (BVASv3and BDCAF)to predict damage, and the influence of immunosuppressive therapy on damage progression, as measured byVDI, in a group of patients with Behcet's Disease. Methods: A study was performed on a cohort of patients diagnosed with Behcet's Disease under surveillance in one tertiary Rheumatology Centre, from a non-endemic area.All documented cases of Behcet's Disease have been diagnosed according to The International Criteria for Behçet's Disease.The Birmingham Activity Score (BVAS)v3, Behcet's Disease Current Activity Form2006 (BDCAF)and Vasculitis Damage index (VDI) were calculated for all patients. Spearman's correlation coefficients were calculated between BVASv3 Score, BDCAF, VDI and immunosuppressive treatment.WindowsExcel/SPSS20.0 has been used to analyse the data.

Results: 20 patients were included in the study, with ages at the time of the 
diagnosis between 13and 60years, $14(70 \%)$ under the age of 40 ,with a male predominance $60 \%$ (12 patients). All patients presented active disease at the time of the diagnosis. In the clinical case series, Spearman's rank correlation coefficient between BVASv3 and BDCAFwas strong $r=0,862$ with $p<0,001$. The outcome analysis after remission was calculated and rank correlation coefficient betweenVDI, and both BVASv3 and BDCAF was moderate (VDI-BVASv3 $r=0,747$, $p<0,001$, VDI- BDCAF $r=0,795, p<0,001)$. As for immunosuppression induction decision and activity scores, the correlation coefficient was moderate $(r=0,734$ for BVASv3, $r=0,647$ for BDCAF)with $p<0,001$. There was a moderate correlation between immunosuppressive treatment and VDI $(r=0,700, p<0,001)$. Since the cause of damage (vasculitis vs. treatment)is not taken into consideration when we calculate VDI, we tried to observe if there are any connections between this and immunosuppression duration. There was a mild correlation and no statistical impact between cyclophosphamide treatment duration and damage calculated as VDI $(r=0,474, p=0,36)$.In contrast, when rank correlation coefficient between cortisone therapy and VDI was calculated, a moderate statistical impact was observed $(r=0,609, p<0,001)$.

Conclusions: Birmingham Vasculitis Activity score (BVAS) v3 and Behcet's Disease Current Activity Form2006 (BDCAF) are reliable tools for evaluating disease activity in patients with Behcet's Disease. They are able to anticipate the need for immunosuppressive therapy and the damage progression, as calculated with Vasculitis Damage Index (VDI).

\section{References:}

[1] Mukhtyar C, Lee R, et al. Modification and validation of the Birmingham vasculitis activity score, Ann Rheum Dis, 2009.

[2] Flossmann O, et al. Development of comprehensive disease assessment in systemic vasculitis. Ann Rheum Dis, 2007.

Disclosure of Interest: None declared

DOI: 10.1136/annrheumdis-2017-eular.4932

\section{THU0556 SALIVARY GLAND ENLARGEMENT IN IGG4-RELATED DISEASE IS ASSOCIATED WITH MULTIORGAN INVOLVEMENT AND HIGHER BASAL DISEASE ACTIVITY}

E. Martin-Nares $^{1}$, A. Angeles-Angeles ${ }^{2}$, G. Hernandez-Molina ${ }^{1}$

${ }^{1}$ Rheumatology; ${ }^{2}$ Pathology, Instituto Nacional de Ciencias Médicas y Nutrición Salvador Zubirán, Mexico City, Mexico

Background: IgG4-related disease (IgG4-RD) is an immune-mediated condition which clinical spectrum encompasses single or multiple organ involvement. Enlargement of major and minor salivary glands is one of the main disease features. Whether salivary gland enlargement is associated with systemic involvement has not been previously evaluated.

Objectives: To elucidate if salivary gland enlargement is associated with systemic disease.

Methods: We included patients with an established diagnosis (definitive: organ involvement, biopsy proven and high lgG4 levels, probable: organ involvement, biopsy proven without high IgG4 levels, possible: organ involvement, high IgG4 levels without histology) of IgG4-RD according to the Comprehensive Diagnostic Criteria, who regularly attend a tertiary referral center in Mexico City (2000-2017). We retrospectively collected demographics, clinical (organ involvement, disease activity and damage assessed by the IgG4-RD Responder Index [IgG4-RD RI] at basal and at 6 months of follow-up, number of relapses, remission and treatment), basal laboratory (C3, C4, ESR, PCR, total eosinophil count, IgG4 levels) as well as imaging and histologic data.

Results: We included 32 patients, 17 (53.1\%) men, mean age $50.2 \pm 14.1$ years and median disease duration 20.5 months. Seven $(21.9 \%)$ have a definitive diagnosis, $12(37.5 \%)$ probable and $13(40.6 \%)$ possible. Overall we identified 21 anatomic sites affected, mainly pancreas $56.2 \%$, lymph nodes $56.2 \%$, lacrimal glands $37.5 \%$ and bile duct $34.3 \%$. Salivary gland involvement was present in $12(37.5 \%)$ patients (2 parotid, 3 minor salivary gland and 7 both). Among these patients, only $5(41.6 \%)$ referred dry mouth and in 7 patients $(58.3 \%)$ glandular enlargement was the onset disease feature. Salivary glandular enlargement was identified only radiologically in 5 patients $(41.6 \%)$ and both clinical and radiologically in $7(58.3 \%)$ patients. When we compared patients with $(n=12)$ vs. without $(n=20)$ salivary gland enlargement, the first group had a higher number of affected organs (6.5 vs. $2, p=0.0001)$ and absolut eosinophil's count (348 vs. $\left.137.5 / \mathrm{mm}^{3}, p=0.05\right)$, a higher prevalence of lacrimal glands $(75 \%$ vs. $15 \%$, $p=0.002)$, lymph nodes $(91.7 \%$ vs. $35 \%, p=0.002)$ and lung involvement $(33.3 \%$ vs. $0 \%, p=0.01)$, azathioprine use $(83.3 \%$ vs. $30 \%, p=0.003)$, as well as a higher basal IgG4-RD RI (12 vs. 6, $p=0.001$ ) and a longer delay in diagnosis (64 month vs. 6.5 months, $p=0.001$ ). We did not find differences regarding gender, age, IgG4 serum levels, C3, C4, ESR, PCR, antinuclear antibodies, rheumatoid factor, anti-Ro/SSA and anti-La/SSB antibodies (negative in all patients), number of relapses, remission at 6 months and damage. We performed a logistic regression analysis (only including the number of organs, the basal IgG4-RD RI and time of follow-up) and found an association of salivary glandular enlargement with the basal IgG4-RD RI (OR 1.63, 95\% Cl 1.12-2.35, p=0.009).

Conclusions: Our study highlights the systemic nature of IgG4-RD. Patients with salivary gland enlargement should be routinely screened for systemic involvement. References:

[1] Carruthers M, Stone J, Deshpande V, et al. Development of an IgG4-RD Responder Index. Int J Rheumatol. 2012;2012:259408.
Acknowledgements: No acknowledgements to report.

Disclosure of Interest: None declared

DOI: 10.1136/annrheumdis-2017-eular.5609

\section{THU0557 VENOUS VESSEL WALL THICKNESS IN LOWER EXTREMITY IS INCREASED IN MALE BEHCET'S DISEASE PATIENTS WITHOUT VASCULAR INVOLVEMENT}

F. Alibaz-Oner ${ }^{1}$, A. Mutis ${ }^{1}$, R. Ergelen ${ }^{2}$, Z. Erturk ${ }^{1}$, T. Ergun $^{3}$, H. Direskeneli ${ }^{1}$. ${ }^{1}$ Rheumatology; ${ }^{2}$ Radiology; ${ }^{3}$ Dermatology, Marmara University, School of Medicine, Istanbul, Turkey

Background: Vascular involvement is seen in up to $40 \%$ of the patients with Behcet's Disease (BD), especially in young males and is one of the major causes of mortality and morbidity. Lower extremity vein thrombosis due to vascular inflammation is the most frequent form of vascular involvement in BD. Recently, assessment of vessel wall thickness (VWT) and venous dilatation by US is suggested to be valuable in patients with vascular inflammation

Objectives: In this study, we investigated whether vessel wall thickness or dilatation is present in young male BD patients prone to venous vascular disease. Methods: Fifteen male patients with BD without major organ involvement followed in Marmara University Behcet's Clinics, 14 healthy male controls and 14 male patients with Ankylosing Spondylitis (AS) were included the study. Bilateral lower extremity venous doppler ultrasonography (US) was performed by an experienced radiologist blinded to cases. No patient was under immunosuppressive treatment. Bilateral common femoral vein (CFV) wall thickness and great/small saphenous vein dilatations were examined. Behçet Syndrome Activity Score (BSAS) was used for the general assessment of disease activity.

Results: The mean disease duration was $9.1 \pm 6.3$ years in patients with BD. BSAS score was $28.9 \pm 19$. Bilateral CFV wall thickness was significantly higher in BD patients compared to healthy controls and $A S(p=0.001, p=0.002$, respectively for right CFV; $p=0.001, p<0.001$, respectively for left CFV) (Table 1). The width of great and small saphenous veins were also higher in patients with $\mathrm{BD}$, but without reaching statistical significance. There were no correlations between BSAS and wall thickness of any vessel.

Table 1. Venous wall measurements of lower extremity in study groups

\begin{tabular}{lcccc}
\hline & $\begin{array}{c}\text { Behçet's } \\
\text { Disease } \\
(\mathrm{n}=15)\end{array}$ & $\begin{array}{c}\text { Healthy } \\
\text { Controls } \\
(\mathrm{n}=14)\end{array}$ & $\begin{array}{c}\text { Ankylosing } \\
\text { Spondylitis } \\
(\mathrm{n}=14)\end{array}$ & P value \\
\hline Age, years & $30.2 \pm 4.5$ & $30 \pm 5.9$ & $30.8 \pm 4.2$ & 0.891 \\
Body Mass Index $\left(\mathrm{kg} / \mathrm{m}^{2}\right)$ & $23.5 \pm 3.5$ & $23.8 \pm 2.8$ & $26.3 \pm 3.8$ & 0.080 \\
Right Common femoral VWT $(\mathrm{mm})$ & $0.69 \pm 0.4$ & $0.26 \pm 0.08$ & $0.28 \pm 0.27$ & $<0.001$ \\
Left Common femoral VWT $(\mathrm{mm})$ & $0.74 \pm 0.4$ & $0.31 \pm 0.13$ & $0.23 \pm 0.13$ & $<0.001$ \\
Right Great saphenous width $(\mathrm{mm})$ & $2.94 \pm 2.6$ & $2.1 \pm 0.71$ & $2.5 \pm 0.73$ & 0.436 \\
Left Great saphenous width $(\mathrm{mm})$ & $3.1 \pm 2.2$ & $2.5 \pm 0.65$ & $2.4 \pm 1.1$ & 0.512 \\
Right Small saphenous width $(\mathrm{mm})$ & $2.4 \pm 1.8$ & $1.4 \pm 0.3$ & $1.7 \pm 0.5$ & 0.126 \\
Left Small saphenous width $(\mathrm{mm})$ & $2.1 \pm 1.5$ & $1.5 \pm 0.8$ & $1.8 \pm 0.6$ & 0.315 \\
\hline
\end{tabular}

VWT: Venous wall thickness.

Conclusions: In preliminary results of our study, an increased venous vessel wall thickness in lower extremity was shown in male BD patients without vascular involvement. As a similar change was not observed in controls, we think, increased VWT might be an early sign of venous inflammation in patients with BD rather than a result of non-specific systemic inflammation. Further studies with a larger group of patients is planned.

Disclosure of Interest: None declared

DOI: 10.1136/annrheumdis-2017-eular.4125

\section{THU0558 THERAPEUTIC RESPONSE TO PREDNISONE ACCORDING TO THE AGE IN POLYMYALGIA RHEUMATICA: A CONTROLLED STUDY}

A. Charpentier ${ }^{1,2}$, F. Verhoeven $^{1}$, M. Sondag ${ }^{1}$, X. Guillot ${ }^{1,2}$, C. Prati ${ }^{1,2}$, D. Wendling ${ }^{1,3} .{ }^{1}$ Rhumatologie, CHRU de Besançon; ${ }^{2}$ Ea 4267 "Pepite "; ${ }^{3} E A$ 4266 "Agents pathogènes et inflammation ", Université de Bourgogne Franche-Comté, Besançon, France

Background: Polymyalgia rheumatica $(P M R)$ is an inflammatory rheumatic disorder which usually affect patients over 65 years old. Different poor prognostic factors are involved in prednisone response including rapid decrease of prednisone dose or female sex. To date, there is no data relating the impact of the age on therapeutic response in PMR

Objectives: The aim of this study was to compare, in case of PMR, the response to prednisone in patients younger than 60 to patients over 65 years old.

Methods: This was a retrospective, monocentric study. We included patients suffering from PMR, meeting ACR 2012 criteria. Patients were classified into two groups, one group with patients less than 60 years, and one group with patients over 65 years. We registered demographic, clinical, biological, and imaging data as well as therapeutic response profile. The local inflammation was evaluated with PET scan, by studying each anatomical site usually affected by PMR. Then, the rate of inflammation was scored from 0 to 3 , according to the intensity of uptake compared to liver. The treatment was standardized. The initial dose of prednisone was of $0.3 \mathrm{mg} / \mathrm{kg} / \mathrm{j}$ during the two first weeks, then, the dose was slowly decreased 